\title{
Grape Seeds Causing Meckel's Diverticulitis, Perforation and Small Bowel Obstruction in a Young Adult Patient: A Case Report and Literature Review
}

Houssam Khodor Abtar', Kassem Jammoul', Mostapha Mneimneh', Rayyan El Lakkis², Mohammad Ahmad Al-Raishouni ${ }^{3}$, Abbass Shibli ${ }^{2}$, Etienne El-Helou ${ }^{2}$, Bilal El Chamaa ${ }^{4}$, Jaafar AlShami , Jad J Terro ${ }^{2}$

${ }^{1}$ Department of General Surgery, Makassed General Hospital, Beirut, Lebanon

${ }^{2}$ Department of General Surgery, Faculty of Medical Sciences at Lebanese University, Beirut, Lebanon

${ }^{3} \mathrm{FAHS}$ surgical services, Michigan, USA

${ }^{4}$ Department of General Surgery, Hotel-Dieu de France University Hospital, Saint-Joseph University, Beirut, Lebanon

Corresponding Author: Jad J Terro, Beirut, Lebanon, j.terro@hotmail.com

Department of General Surgery, Makassed General Hospital, Beirut, Lebanon.

Financial support: None

Conflict of Interest: None

\begin{abstract}
Background: Meckel's diverticulum is a true diverticulum consisting of a 3-layered outpouching of the bowel wall along the antimesenteric border. It is a remnant of the omphalomesenteric duct and the most common congenital gastrointestinal disorder. It has a male predilection and remains asymptomatic in the majority of cases. It constitutes a diagnostic challenge to physicians, as it can present with gastrointestinal bleeding in the pediatric population, and as an intestinal obstruction in adults. While the management of an asymptomatic Meckel's diverticulum is on a case-by-case basis, when symptomatic, prompt surgical intervention is necessary, and a laparoscopic approach allows both in-situ diagnosis and treatment.

Case Report: A 23-year-old previously healthy female patient, presented with diffuse abdominal pain, vomiting, and obstipation. Abdominal X-Ray and abdominopelvic Computed Tomography showed an intra-abdominal inflammatory process and evidence of bowel obstruction but were not conclusive. The patient was admitted to the hospital for management, and on the third day of hospitalization physical examination showed abdominal guarding suggestive of peritonitis. An urgent exploratory laparotomy identified a Meckel's Diverticulum obstructed with phytobezoar grape seeds, and an inflamed and perforated bowel wall, with adhesive bands to proximal small bowel loops, necrosis, and resultant small bowel obstruction. We resected the Meckel's diverticulum and the necrotic bowel and performed an end-to-end primary anastomosis of the small bowel. The postoperative course was uneventful, and the patient was discharged on the fourth postoperative day.

Conclusion: The diagnosis of Meckel's diverticulum remains a challenge as it has a myriad of clinical presentation and radiological imaging sometimes fails to provide a definite diagnosis. It must be systematically included in the differential diagnosis of small bowel obstruction in adult patients, as it requires prompt surgical intervention for both diagnosis and treatment.
\end{abstract}

Keywords: Meckel's diverticulum, small bowel obstruction, meckel's diverticulitis, perforation, diverticulitis, phytobezoar, diagnostic challenge, case report 


\section{Background}

Meckel's diverticulum (MD) is the most frequent gastrointestinal (Gl) congenital disorder, with an incidence rate of $1-4 \%$ [1]. It is famous for the "rule of $2 \mathrm{~s}$ ": presenting mostly under the age of 2 , within 2 feet from the ileocecal valve, occurring in males twice more than in females, and affecting $2 \%$ of the general population [1]. It is a true antimesenteric diverticulum comprised of the 3 layers of a normal intestinal wall. It occurs due to the persistence of the embryologic omphalomesenteric duct and has its innate blood supply by the omphalomesenteric artery. Knowing it is mostly asymptomatic in adults, MD can present, when complicated, with non-specific signs and symptoms mimicking other visceral abnormalities such as appendicitis and inflammatory diseases [1, 2]. Patients seeking medical attention are most commonly below the age of 10 and usually present with gastrointestinal bleeding, while adults present mainly with symptoms of intestinal obstruction [3].

Although rare, the most common cause of MDinduced obstruction is intussusception and volvulus. Phytobezoars are even rarer as a cause of MD-induced bowel obstruction [4]. Other uncommon complications in adults include inflammation or diverticulitis with perforation, neoplasm formation, and fistulas [3]. To this day, MD remains a diagnostic challenge. Various types of imaging techniques have been used to detect it preoperatively, however, they often fail and surgical exploration remains a means of definitive diagnosis and treatment $[5,6]$. We present the case of a young female patient diagnosed, intraoperatively, with Meckel's diverticulitis caused by grape seeds phytobezoar, leading to perforation and subsequent small bowel obstruction by inflammatory adhesions.

\section{Case Presentation}

A previously healthy 23-year-old female patient, with no previous surgical procedures, presented with a 4-day history of diffuse pain in her abdomen. She described her pain as sudden in onset, diffuse, intermittent, and colicky, with an intensity of 5/10, and reported obstipation, and many episodes of non-bilious, non-feculent vomiting. She denied any weight loss. She looked ill and weak, had a BMl of $22.5 \mathrm{~kg} / \mathrm{m}^{2}$, with a weight of $56.3 \mathrm{Kg}$ and a height of $158 \mathrm{~cm}$. The abdominal examination showed a soft but distended and diffusely tender abdomen with hyperresonance. A digital rectal exam showed an empty rectum with no gross evidence of lower gastrointestinal bleeding. She was hemodynamically stable and afebrile, with normal hemoglobin, creatinine, electrolytes, and no leukocytosis.

An upright plain kidney, ureter, and bladder (KUB) $\mathrm{X}$-ray showed several air-fluid levels, distended small bowels, and an absence of air in the rectum (Fig.1). An urgent abdominopelvic computed tomography (CT) scan with an intravenous (IV) and per-os (PO) contrast showed a distended small bowel and stomach, with multiple air-fluid levels and a transition point at the level of the small bowel. There was also intestinal wall thickening along with fat stranding around an intestinal loop, as well as fluid in the Douglas pouch (Fig. 2-3). The appendix was normal.

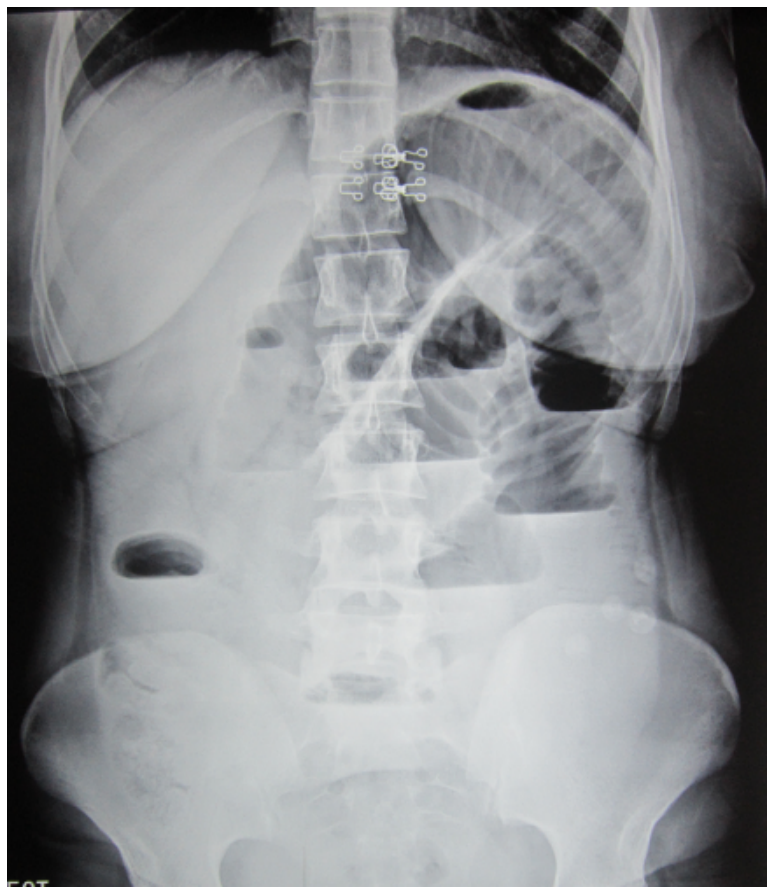

Figure 1: KUB showing several air-fluid levels, distended small bowels, and an absence of air in the rectum

We placed a nasogastric tube for decompression and drainage and started the patient on broadspectrum antibiotics (piperacillin/tazobactam 4.5 grams given intravenously every 6 hours and metronidazole 500 milligrams intravenously every 8 hours) for her unknown abdominal inflammatory process. On the second day, we consulted a gastroenterologist who recommended a colonoscopy to rule out inflammatory bowel disease. The colonoscopy showed no significant findings. On her third hospitalization day, the patient's status deteriorated. Her laboratory analysis was significant for leukocytosis, and her physical examination revealed diffuse abdominal guarding, suggestive of peritonitis. She remained hemodynamically stable, but we opted for urgent exploratory laparotomy. With the patient in a supine position, we performed a 
midline xiphopubic laparotomy that revealed an abdomen full of purulent material and fibrous adhesions. Exploration showed a collection of inflamed tissue pulling the ileal loop to the anterior abdominal wall. Dissection of this collection showed a perforated $8.5 \mathrm{~cm}$ MD containing a phytobezoar, in which we identified undigested grape seeds. This MD had resulted in small bowel obstruction with necrosis of the proximal $50 \mathrm{~cm}$ segment of bowels (Fig. 3).

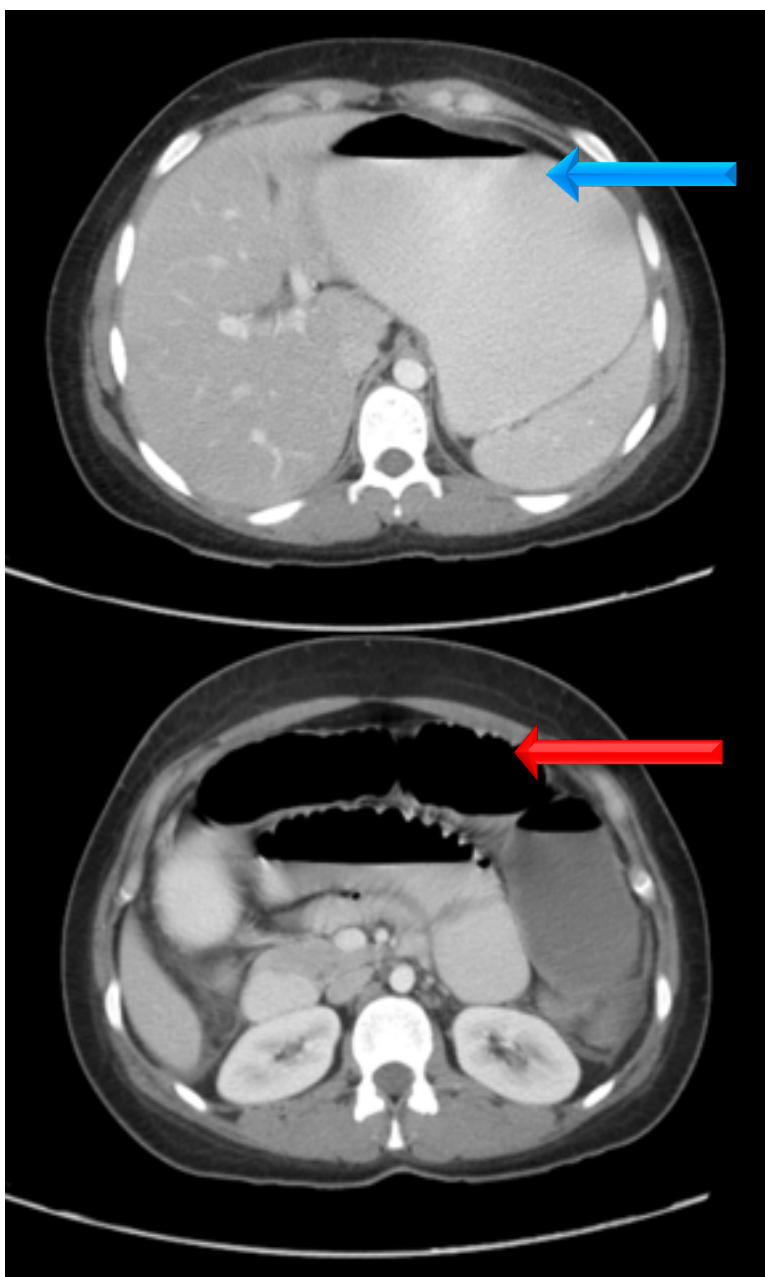

Figure 2: Abdominopelvic CT scan showing the distended stomach (blue arrow) and small bowels (red arrow)

We resected the necrotic ileal loop with a $45 \mathrm{~mm}$ Endo GIA and performed a side-to-side primary anastomosis. We copiously irrigated the cavity with normal saline and placed a Pezzer catheter in the pouch of Douglas. We closed the wound layer by layer and did a wound dressing using a $4 \times 4$ inch gauze. We tagged the specimens (Fig. 4) and sent them to pathology for gross and microscopic evaluation. Histopathology showed a neutrophilic inflammatory reaction causing necrosis on the bowel segment and a Meckel's diverticulum with eroded intestinal mucosa containing grape seeds. The patient had a smooth postoperative course and returned home on day 4 post-operation, after tolerating a soft diet.

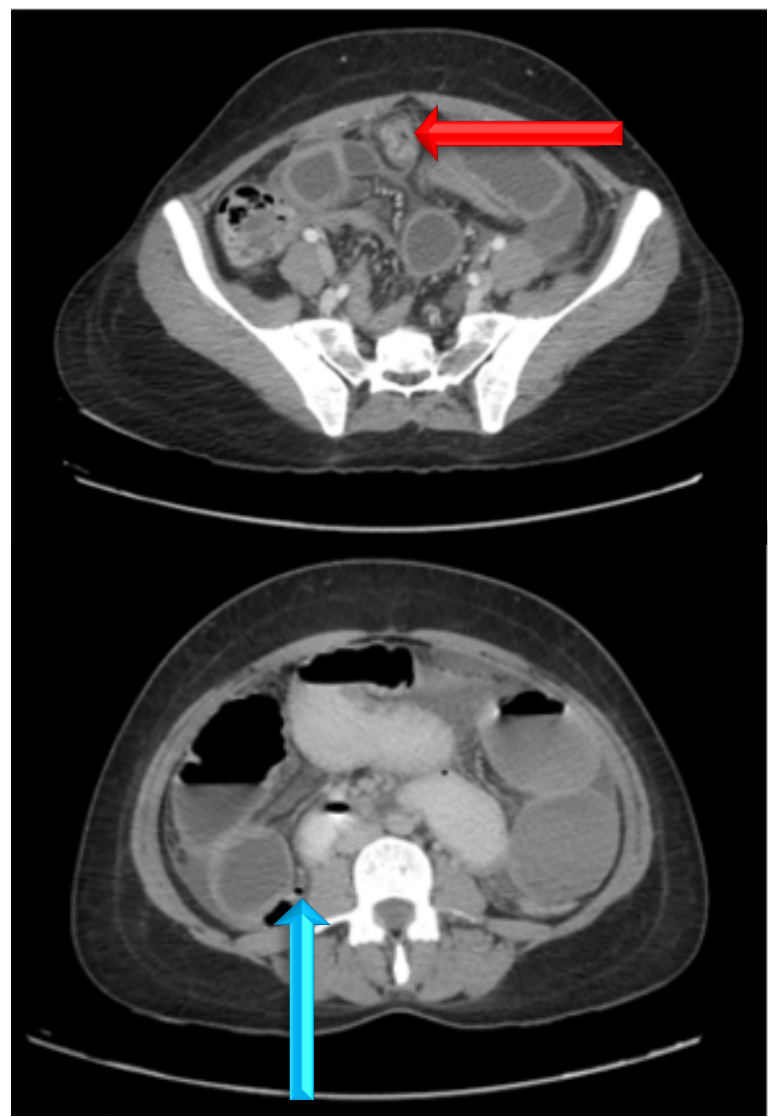

Figure 3: Abdominopelvic CT scan with IV and PO contrast showing a normal aerated appendix (red arrow) and an inflamed bowel loop with fat stranding and marked inflammation of the bowel walls (blue arrow).

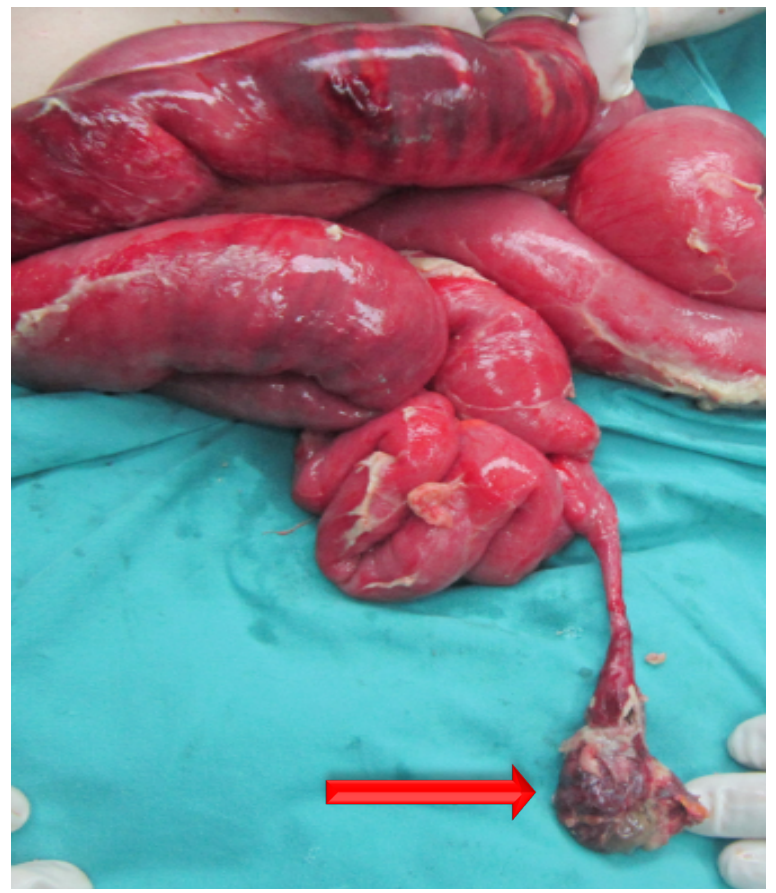

Figure 4: An intraoperatively taken photograph of the inflamed Meckel's Diverticulum (red arrow) affecting a distant small bowel loop 


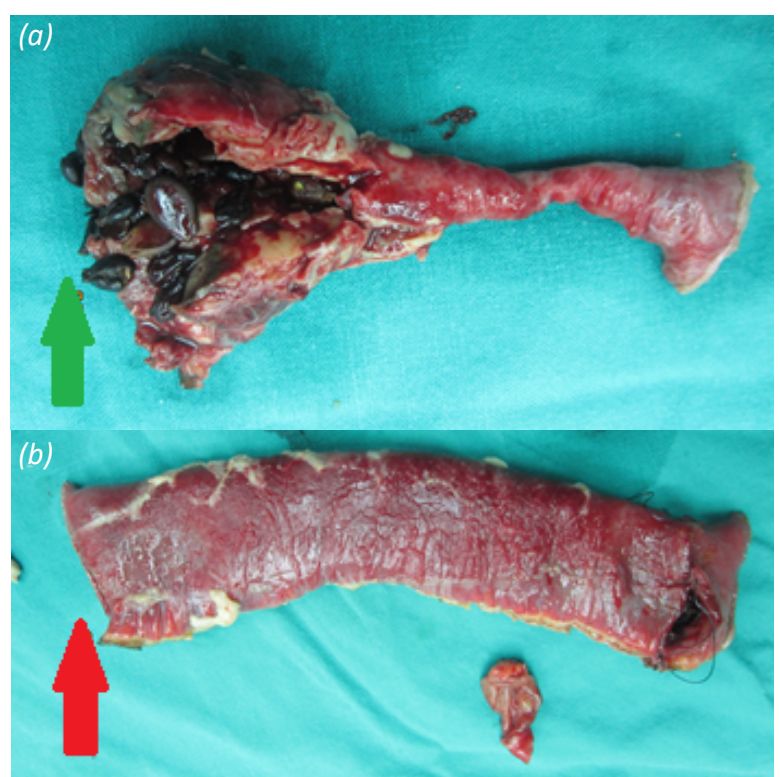

Figure 5: Specimens sent to pathology (a) the stapled resection of the Meckel's Diverticulum with Grape seeds inside (green arrow) and (b) the resected small bowel segment (red arrow).

\section{Discussion}

Meckel's Diverticulum was named by Dr. Johann Friedrich Meckel in 1809 and described in 1958 by Dr. Fabricius Hildanus [1]. To this day, MD remains a challenging medical diagnosis due to its nonspecific symptomatology, which, in 5 to $17 \%$ of cases, depends on the developed complications [7]. These complications mostly occur in patients younger than 10 years old $(40 \%)$, and decrease with age, reaching $0 \%$ at 76 years old [4]. Male patients have a higher complication rate than females. In adults, intestinal obstruction accounts for up to $50 \%$ of the complications and is mainly due to intussusception, with the MD serving as a lead point for intestinal telescoping [3]. Additional uncommon causes of obstruction include volvulus secondary to umbilical fibrous bands, diverticular strictures, Litter's hernia, and inflammatory adhesions. Besides obstruction, another complication is the inflammation of the diverticula, or Meckel's diverticulitis, which resembles, in its presentation and pathophysiology, acute appendicitis, rendering a definite diagnosis challenging without direct surgical examination [3].

Uncommon complications in adults include hemorrhage, perforation, and rarely, fistula formation and malignant transformation [3]. In pediatric populations, the most common complication is hemorrhage (50\%). It presents as lower Gl bleeding and is frequently due to heterotopic mucosa, which is, in $90 \%$ of the cases, ectopic gastric tissue without Helicobacter pylori infection [2, 3]. It is relatively uncommon to have several complications occurring together including Meckel's diverticulitis, perforation, and Small Bowel Obstruction (SBO) due to the inflammatory process, as was the case in our patient. Furthermore, diverticulitis mostly occurs due to the acidic secretions of the ectopic gastric mucosa rather than due to enterocoliths, tumors, or foreign bodies that obliterate the diverticulum and result in inflammation, necrosis, and perforation [3, 6]. Enterocolith formation as in our patient is only seen in 3-10\% of MDs [6].

Only one similar case to ours was reported in the literature in 1958, making grape seeds a very rare cause of MD obstruction, diverticulitis, and perforation [8]. However, despite popular belief, seeds are not associated with an increased risk of diverticulitis and perforation [9]. Risk factors such as delayed gastric emptying, malabsorption, and consumption of high fiber diet are well known for the development of phytobezoars in adults. [8]

The diagnosis of MD remains challenging for radiologists. Unfortunately, less than $10 \%$ of symptomatic cases of $M D$ are preoperatively identified [10]. Conventional radiography is of limited value and may be limited to signs of bowel obstruction, air-fluid level in the diverticulum, or air under the diaphragm. Sometimes, it can show the enterocolith. Small bowel contrast studies have variable value in terms of MD diagnosis. These contrast studies may show the classical appearance of the antimesenteric diverticulum, along with the irregularity of the mucosa inside the diverticulum in cases of gastric ectopic mucosa or intra-diverticular hemorrhage. In such studies, enteroclysis small bowel enema is more sensitive than traditional barium swallow. Ultrasound and conventional CT scans for the abdomen and pelvis are not the best options for MD diagnosis, and their importance is limited to complicated MD. Ultrasound can show a blind end sac at the right lower abdominal quadrant, irregular ectopic gastric mucosa, if present, and enterocoliths leading to shadowing during the sonographic examination. CT-scan can localize the enterocoliths, position them relative to the ileocecal valve, and rule out appendicitis. CT with enteroclysis has an added value over the conventional CT scan by showing the MD lumen and mucosa. Mesenteric angiography can show two entities, the extravasation of contrast material through a bleeding MD, and/or the abnormally patent omphalomesenteric artery in cases of nonbleeding MD. 99mTc-Na-pertechnetate scintigraphy has an advantage in detecting MD 
that has ectopic gastric mucosa in $15-50 \%$; its sensitivity is higher in pediatric populations $(85 \%)$ than in adult populations (58\%). The emerging technology of Wireless capsule endoscopy can allow us to see the MD hemorrhage that can be missed in scintigraphy and angiography, leading to a higher diagnostic yield and promising outcomes $[6,11]$.

With a complication rate reaching $6 \%$, the management of incidental MD remains debatable. Resection of an uncomplicated MD is recommended in the presence of any of the following criteria: 1) age younger than 50 years, 2) narrow-based diverticula with a diverticular length more than $2 \mathrm{~cm}, 3$ ) Presence of a fibrous band between the diverticulum and the umbilicus, and 4) Presence of ectopic gastric tissue with the diverticulum. All these criteria dramatically increase the risk of complications [2, 12]. If one criterion is present, the rate of complication is $17.2 \%$, increasing to $25.3 \%$ if a second one is added, to $42 \%$ if a third one is present, and reaching $70 \%$ if all four are present [12].

A laparoscopic approach is preferred over an open laparotomy as it allows for a complete diagnostic evaluation and carries a lower morbidity and mortality rates [5]. Surgical techniques include simple stapled transverse diverticulectomy for non-bleeding MD longer than two centimeters; wedge-shaped resection for broad-based non-bleeding MD and segmental bowel resection in cases of a bleeding MD, a wide-based MD with ectopic mucosa, an inflamed or perforated base, and/or MD malignancy. It is important to confirm the complete resection of malignancy or residual ectopic tissue by frozen sections since this ectopic tissue is a hotspot carrying 70 times more risk to cancer. Finally, small bowel running is important in cases of complicated MD, especially if the appendix is normal $[1,7,13,14]$.

This was the case of a young female patient with an obstructed and inflamed MD leading to perforation and SBO due to an inflammatory adhesive process. It was successfully treated with laparotomy, simple-stapled diverticulectomy technique because of a longer than $2 \mathrm{~cm} \mathrm{MD}$ without pathologic base, and with distant small bowel segmental resection due to inflammation and necrosis secondary to the MD inflammatory process. Surprisingly, grape seeds acting as phytobezoars were seen upon emptying the MD, thus resulting in this pathologic picture, making it the second reported case of its type in English literature [8].

\section{Conclusion}

Leading the congenital gastroenterology malformations, $M D$ is an interesting and challenging finding for pediatric surgeons, general surgeons, and radiologists. It can mimic many abdominal pathologies, and differentiation is rarely successful preoperatively. Every imaging diagnostic technique has a given value, with scintigraphy being the best in most cases. Due to its complication rate which decreases with age, incidental MD can be resected on a case-by-case basis, however, symptomatic and complicated MDs must always be excised. Different surgical techniques are present depending on the given scenario, and laparoscopy is playing a chief role. Therefore, a higher level of suspicion for MD diagnosis and better implementation of diagnostic techniques must be considered.

\section{References}

1. Gonçalves A, Almeida M, Malheiro L, Costa-Maia J. Meckel's diverticulum perforation by a fish bone: A case report. Int J Surg Case Rep. 2016;28:237-240. doi: 10.1016/j.ijscr.2016.08.026. Epub 2016 Sep 7. PMID: 27744259; PMCID: PMC5066196. https://doi.org/10.1016/j.ijscr.2016.08.026

2. Bani-Hani KE, Shatnawi NJ. Meckel's diverticulum: comparison of incidental and symptomatic cases. World $\mathrm{J}$ Surg. 2004 Sep;28(9):917-20. doi: 10.1007/s00268-0047512-3. PMID: 15593467. https://doi.org/10.1007/s00268-004-7512-3

3. Dumper J, Mackenzie S, Mitchell P, Sutherland F, Quan $\mathrm{ML}$, Mew D. Complications of Meckel's diverticula in adults. Can J Surg. 2006 Oct;49(5):353-7. PMID: 17152574 ; PMCID: PMC3207587.

4. Cartanese C, Petitti T, Marinelli E, Pignatelli A, Martignetti D, Zuccarino M, Ferrozzi L. Intestinal obstruction caused by torsed gangrenous Meckel's diverticulum encircling terminal ileum. World J Gastrointest Surg. 2011 Jul 27;3(7):106-9. doi: 10.4240/wjgs.v3.i7.106. PMID: 21860699; PMCID: PMC3158886. https://doi.org/10.4240/wjgs.v3.i7.106

5. Ding Y, Zhou Y, Ji Z, Zhang J, Wang Q. Laparoscopic management of perforated Meckel's diverticulum in adults. Int J Med Sci. 2012;9(3):243-7. doi: 10.7150/ijms.4170. Epub 2012 May 4. PMID: 22577339; PMCID: PMC3348529. https://doi.org/10.7150/ijms.4170

6. Elsayes KM, Menias CO, Harvin HJ, Francis IR. Imaging manifestations of Meckel's diverticulum. AJR Am J Roentgenol. 2007 Jul;189(1):81-8. doi: 10.2214/AJR.06.1257. $\quad$ PMID: 17579156. https://doi.org/10.2214/AJR.06.1257

7. Piñero A, Martínez-Barba E, Canteras M, Rodriguez JM, Castellanos G, Parrilla P. Surgical management and complications of Meckel's diverticulum in 90 patients. Eur J Surg. 2002;168(1):8-12. doi: 10.1080/110241502317307508. PMID: 12022375. https://doi.org/10.1080/110241502317307508 
8. Rumore A. Perforation of Meckel's diverticulum by grape seeds. Med J Aust. 1958 Jan 25;45(4):110-2. PMID: 13515835.

https://doi.org/10.5694/j.1326-

5377.1958.tb86083.x

9. El-Sayed C, Radley S, Mytton J, Evison F, Ward ST. Risk of Recurrent Disease and Surgery Following an Admission for Acute Diverticulitis. Dis Colon Rectum. 2018 Mar;61(3):382-389.

doi: 10.1097/DCR.0000000000000939. PMID: 29420430. https://doi.org/10.1097/DCR.0000000000000939

10. Modi S, Kanapathy Pillai S, DeClercq S. Perforated Meckel's diverticulum in an adult due to faecolith: A case report and review of literature. Int $\mathrm{J}$ Surg Case Rep. 2015;15:143-5. doi: 10.1016/j.jjscr.2015.07.029. Epub 2015 Aug 22. PMID: 26363105; PMCID: PMC4601945. https://doi.org/10.1016/j.ijscr.2015.07.029

11. Thurley PD, Halliday KE, Somers JM, AI-Daraji WI, Ilyas M, Broderick NJ. Radiological features of Meckel's diverticulum and its complications. Clin Radiol. 2009 Feb;64(2):109-18. doi: 10.1016/j.crad.2008.07.012. Epub 2008 Sep $25 . \quad$ PMID: 19103339 https://doi.org/10.1016/j.crad.2008.07.012

12. Robijn J, Sebrechts E, Miserez M. Management of incidentally found Meckel's diverticulum a new approach: resection based on a Risk Score. Acta Chir Belg. 2006 JulAug;106(4):467-70.

doi: 10.1080/00015458.2006.11679933. PMID: 17017710. https://doi.org/10.1080/00015458.2006.11679933

13. Erol V, Yoldaş T, Cin S, Çalışkan C, Akgün E, Korkut M. Complicated Meckel's diverticulum and therapeutic management. Ulus Cerrahi Derg. 2013 Jun 1;29(2):63-6. doi: 10.5152/UCD.2013.36. PMID: 25931848; PMCID: PMC4379835. https://doi.org/10.5152/UCD.2013.36

14. Sharma RK, Jain VK. Emergency surgery for Meckel's diverticulum. World J Emerg Surg. 2008 Aug 13;3:27. doi: 10.1186/1749-7922-3-27. PMID: 18700974; PMCID: PMC2533303. https://doi.org/10.1186/1749-7922-3-27 